\title{
Activation of peroxisome proliferator activated receptor alpha ameliorates ethanol induced steatohepatitis in mice
}

Lingbo Kong ${ }^{1}$, Weiguang Ren ${ }^{1}$, Wencong $\mathrm{Li}^{1}$, Suxian Zhao ${ }^{1}$, Hongmei $\mathrm{Mi}^{1}$, Rongqi Wang ${ }^{1}$, Yuguo Zhang ${ }^{1}$, Wenjuan $\mathrm{Wu}^{1}$, Yuemin $\mathrm{Nan}^{1 *}$ and Jun $\mathrm{Yu}^{2}$

\begin{abstract}
Background: Peroxisome proliferator activated receptor alpha (PPAR $\alpha$ ) regulates lipids metabolism and inhibits inflammatory response. However, the role of PPAR $\alpha$ in alcoholic liver disease is largely unknown. We aim to elucidate the effect and the molecular basis of PPAR $\alpha$ in ethanol induced hepatic injury in mice.

Results: C57BL/6J mice fed with 4\% ethanol-containing Lieber-DeCarli liquid diet for 12 weeks exhibited hepatocyte steatosis, necrosis and inflammatory infiltration, accompanied with elevated serum alanine aminotransferase (ALT) and aspartic transaminase (AST) levels, decreased hepatic expression of PPAR $\alpha$, lipids oxidation promoting genes and antiinflammatory factors, as well as enhanced hepatic expression of fatty acids synthesis promoting genes and proinflammatory cytokines. Induction of PPAR $\alpha$ by PPAR $\alpha$ agonist WY14643 treatment for 2 weeks ameliorated the severity of liver injury and restored expression of genes altered by ethanol treatment. However, administration of PPAR $\alpha$ antagonist GW6471 for 2 weeks promoted the inflammatory response.
\end{abstract}

Conclusions: The present study provided the evidence for the protective role of PPAR $\alpha$ in ameliorating ethanol induced liver injury through modulation of the genes related to lipid metabolism and inflammatory response.

Keywords: Peroxisome proliferator activated receptor alpha, ethanol, steatohepatitis, animal experiment

\section{Background}

Alcoholic liver injury is a progressive process encompassing hepatic steatosis and steatohepatitis. The latter may progress to liver fibrosis, cirrhosis and even hepatocellular carcinoma [1]. Chronic ethanol exposure impairs fatty acid oxidation and enhances lipogenesis by targeting key transcriptional regulators of genes controlling these metabolic processes, including peroxisome proliferators activated receptor gamma coactivator 1 alpha (PGC-1 $\alpha)$ [2], sterol regulatory element binding protein 1 (SREBP-1) and its downstream genes, such as fatty acid synthase (FAS) [2], resulting in the accumulation of triglyceride in the liver (steatosis). Fat accumulation renders the liver more susceptible to other injuries. Ethanol also contributes to the up-regulation of pro-inflammatory factors, osteopontin

\footnotetext{
* Correspondence: nanyuemin@163.com

'Department of Traditional and Western Medical Hepatology, Third Hospital of Hebei Medical University, Shijiazhuang, China

Full list of author information is available at the end of the article
}

(OPN) [3-5] and cyclooxygenase-2 (COX-2) [6] in the liver, which promotes inflammatory injury and causes alcoholic steatohepatitis. Pharmacological treatment for patient with alcoholic steatohepatitis is still not available. There is compelling need to identify agent to protect liver against ethanol-related inflammatory injury.

Peroxisome proliferator activated receptor alpha (PPAR $\alpha$ ), interacts with the retinoid $\mathrm{X}$ receptor to function as a transcription factor to induce the expression of a series of genes involved in fatty acid transport, mitochondrial fatty acid oxidation, catabolism, and inflammatory responses [7-11]. Down-regulation and/or dysfunction of PPAR $\alpha$ are involved in the development of ethanol induced liver injury [11]. However, the role of PPAR $\alpha$ in pathogenesis of alcoholic liver disease (ALD) remains largely unknown. In this study, we investigated the effects of PPAR $\alpha$ activation in evolution of alcoholic steatohepatitis and the molecular basis of its action in animal experiments.

\section{C) Biomed Central}




\section{Results}

Activation of PPAR $\alpha$ by WY14643 lowered the serum levels of alanine aminotransferase (ALT) and aspartic transaminase (AST) in mice fed with ethanol liquid diet As shown in Figure 1, mice fed with 4\% ethanolcontaining Lieber-DeCarli liquid diet for 12 weeks showed significantly higher serum ALT and AST levels $(P<0.001)$ compared with Control group, indicating hepatic injury. A significant reduction of serum ALT and AST levels $(P<0.001)$ was noticed after WY14643 treatment. However, GW6471 treatment further elevated the ALT level $(P<0.01)$ than those fed ethanol liquid diet only (Figure 1).

Activation of PPAR $\alpha$ ameliorated liver injury in mice fed with ethanol liquid diet

The liver sections from mice fed with ethanol-containing liquid diet exhibited disordered lobule structure, hepatocyte ballooning, moderate steatosis, inflammatory infiltration and mild hepatocyte necrosis. WY14643 significantly ameliorated hepatic steatosis and inflammation $(P<0.001)$ (Figure 2).

\section{Activation of PPAR $\alpha$ improved ultrastructural damage of} hepatocytes in mice fed with ethanol liquid diet

Rich organelles including mitochondria, endoplasmic reticulum and ribosomes are observed in hepatocytes of normal control livers under electron microscopy. Whilst, in the liver sections of ethanol feeding mice, mitochondrial damage in hepatocytes with broken cristae, ruptured membranes and merged cristae/membranes are observed. Granule fusion and degranulation phenomenons are also found in rough endoplasmic reticulum. These ultrastructural changes in hepatocytes are clearly improved by WY14643 administration (Figure 2C).

\section{Hepatic expression of PPAR $\alpha$ and PPAR $\alpha$-responsive genes in mice fed with ethanol liquid diet and treated with WY14643 or GW6471}

As shown in Figure 3, hepatic expression of PPAR $\alpha$ mRNA $(P<0.05)$ and protein $(P<0.05)$, as well as
PPAR $\alpha$-responsive genes cytochrome P450 4A10 (CYP4A10) $(P<0.001)$ and CYP4A14 $(P<0.01)$ mRNA was down-regulated by ethanol. WY14643 administration increased the expression levels of PPAR $\alpha(P<0.05)$, CYP4A10 $(P<0.001)$ and CYP4A14 $(P<0.001)$. However, GW6471 reduced PPAR $\alpha(P<0.05)$ and CYP4A14 $(P<$ $0.001)$ mRNA expression.

\section{Induction of PPAR $\alpha$ regulates hepatic expression of lipid} metabolism related genes

To seek an explanation for the ameliorated hepatic steatosis under WY14643 administration, we assessed the hepatic expression levels of genes involved in lipid metabolism. Relative to control mice, hepatic FAS mRNA $(P<0.001)$ and protein $(P<0.001)$ were enhanced, while sirtuin 1 (SIRT1) mRNA $(P<0.001)$ and protein $(P<0.001)$ as well as PGC-1 $\alpha$ protein $(P<0.01)$ were reduced in mice fed with ethanol liquid diet. Administration of WY14643 increased mRNA and protein expressions of fibroblast growth factor 21 (FGF21) $(P<0.001$ and $P<0.01$, respectively), SIRT1 $(P<0.05$ and $P<0.01)$, PGC- $1 \alpha(P<0.001$ and $P<0.001)$, down-regulated FAS expression $(P<0.001$ and $P<0.05)$ as compared with mice fed ethanol liquid diet only (Figure 4). GW6471 decreased the FGF21 mRNA $(P<0.001)$ and protein $(P<0.05)$ expression, while increased FAS mRNA $(P<0.01)$ expression in mice fed with ethanol.

\section{Activation of PPAR $\alpha$ suppressed hepatic expression of pro-inflammatory factors}

We further evaluated the role of PPAR $\alpha$ in the development of ethanol induced liver injury by assessing the hepatic expression levels of pro-inflammatory factors phosphatidylinositol 3-kinase (PI3K), OPN and COX-2. We found that ethanol increased the expression of PI3K, OPN and COX-2. Mice treated with WY14643 showed significantly reduced hepatic mRNA and protein expression for PI3K $(P<0.001$ and $P<0.01$, respectively), OPN $(P<0.001$ and $P<0.001)$ and COX-2 $(P<0.01$ and $P<$ $0.05)$ as compared with mice fed with ethanol liquid diet only (Figure 5). On the other hand, GW6471 further
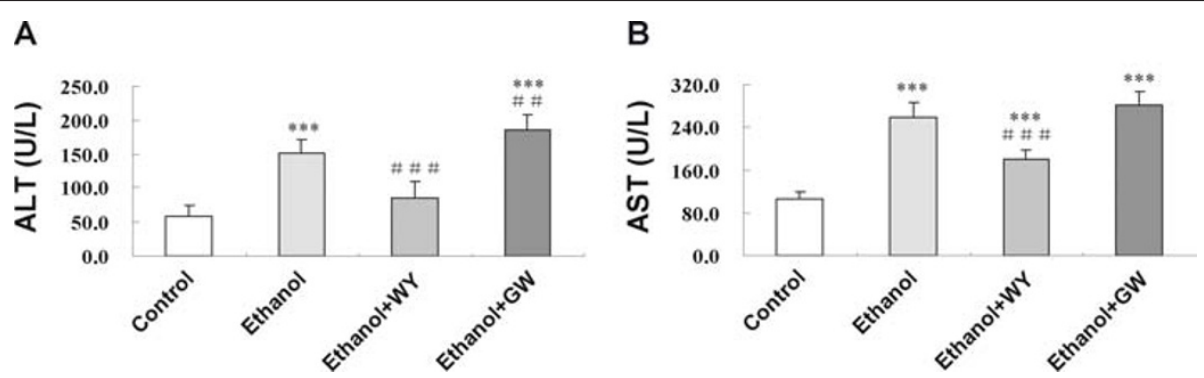

Figure 1 Effects of ethanol with treatment of WY14643 or GW6471 on serum (A) ALT level and (B) AST level. Data are expressed as the mean $\pm S D$ ( $n=6$ per group). ${ }^{* * *} P<0.001$ compared with Control group; ${ }^{\# \#} P<0.01$, ${ }^{\# \#} P<0.001$ compared with Ethanol group. 


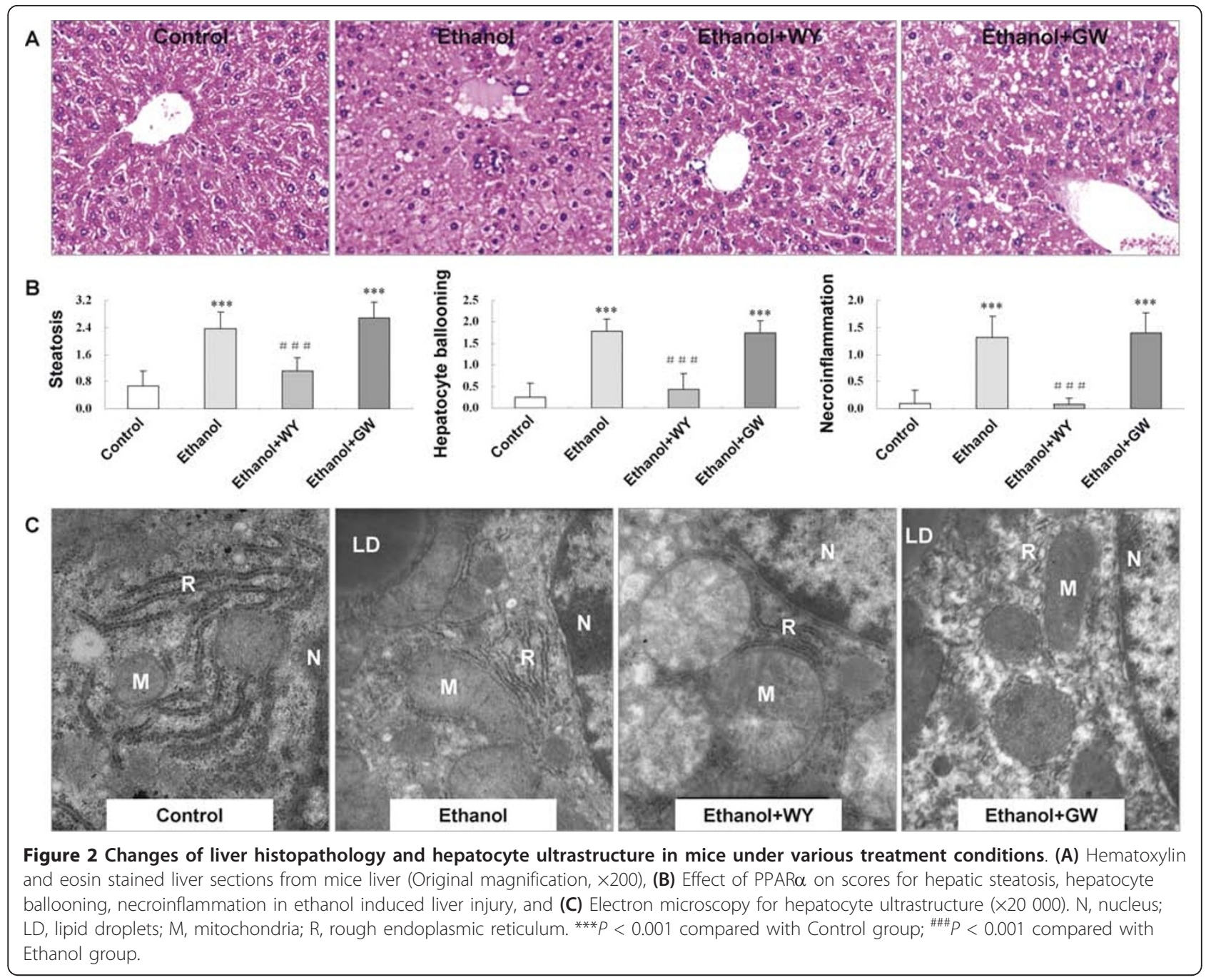

increased PI3K protein $(P<0.05)$ and COX-2 mRNA $(P<$ $0.05)$ expression, but didn't affect OPN expression.

\section{Activation of PPAR $\alpha$ enhanced hepatic expression of adiponectin and heme oxygenase-1 ( $\mathrm{HO}-1$ )}

Hepatic mRNA and protein expression of adiponectin, a major anti-inflammatory adipokine, and $\mathrm{HO}-1$, a key antioxidative stress factor, were evaluated by RT-PCR and Western blot. WY14643 up-regulated mRNA and protein expression for adiponectin $(P<0.001$ and $P<0.01$, respectively) and for HO-1 $(P<0.001$ and $P<0.001)$ in mice fed with ethanol liquid diet (Figure 6). GW6471 didn't alter expression of these two genes (Figure 6).

\section{Discussion}

C57BL/6J mice fed with $4 \%$ ethanol-containing LieberDeCarli liquid diet for 12 weeks showed hepatic steatosis, hepatocyte ballooning, inflammatory cell infiltration and hepatocyte necrosis, accompanied with elevated serum ALT and AST levels. Under transmission electron microscopy, we found that mitochondrial cristae were broken, the membranes were ruptured, and most of the cristae were merged with parts of the membranes. These results indicated that this ALD mouse model mimicked the human alcoholic liver injury.

PPAR $\alpha$, a member of the nuclear hormone receptor superfamily and a receptor for free fatty acid (FFA) [12-14], represses hepatic lipid accumulation by regulating the expression of genes involved in fatty acid synthesis, oxidation and storage. It is also inhibited inflammatory response via inhibiting transcription of nuclear factor- $\kappa \mathrm{B}$ and production of inflammatory cytokines [11]. In this study, we found PPAR $\alpha$ expression was down-regulated accompanied by the development of ethanol induced hepatic injury. Induction of PPAR $\alpha$ by WY14643 treatment for 2 weeks attenuated ethanol induced liver injury, as evidenced by diminished histological steatosis and inflammatory response, improved hepatocyte ultrastructure, as well as 


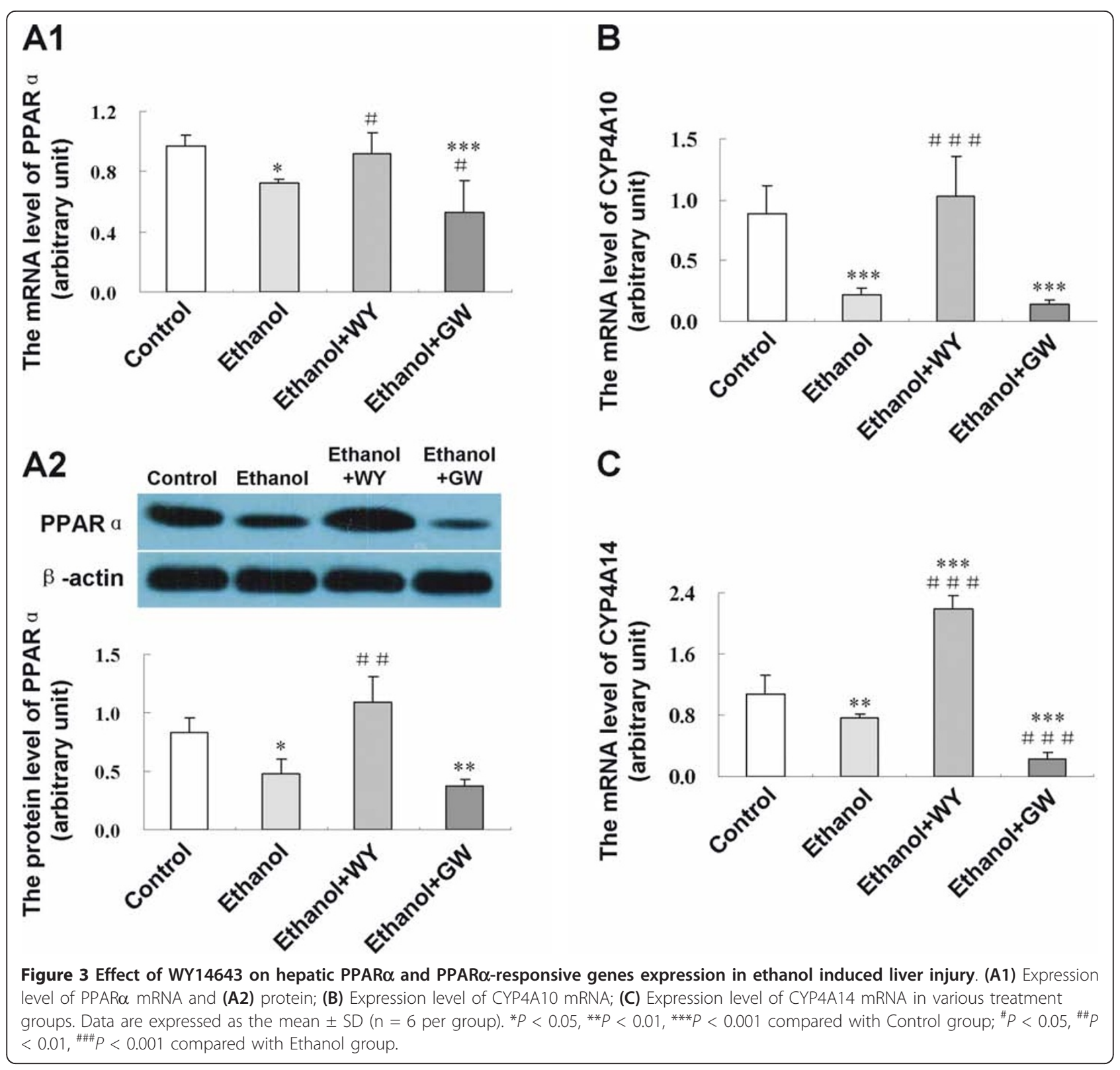

decreased serum ALT and AST levels. Administration of PPAR $\alpha$ antagonist GW6471 for 2 weeks increased hepatic inflammation. These results indicated that PPAR $\alpha$ might play an important protective role in the pathogenesis of ALD.

We further explored the potential mechanisms of the protective effect of PPAR $\alpha$ by the use of selective agonist WY14643. Ethanol and its metabolic products impair tricarboxylic acid cycle activity and fatty acid $\beta$-oxidation in the mitochondria, resulting in FFA overload and triglyceride accumulation in the liver, ultimately leading to hepatic steatosis. Characteristic PPAR $\alpha$ target genes CYP4A10 and CYP4A14 are involved in fatty acid oxidation in a PPAR $\alpha$-dependent manner [15]. Hepatic CYP4A10 and
CYP4A14 mRNA expression could be reduced by ethanol and restored by WY14643. FGF21, a hormone primarily produced by liver and fat tissue, was reported to improve lipid metabolism [16-18]. In this study, hepatic FGF21 expression was enhanced by WY14643. In addition, the metabolic effect of FGF21 depends on the activation of its downstream signaling cascade involving the SIRT1 axis [16-18]. The latter had been reported to be responsible for PGC- $1 \alpha$ activation and SREBP-1 reduction in the liver $[19,20]$. We found that WY14643 restored the hepatic expression of SIRT1 which was reduced by alcohol administration in mice. Induction of SIRT1 enhanced mitochondrial oxidative capacity as demonstrated by increases in oxygen consumption and citrate synthase activity, as well 


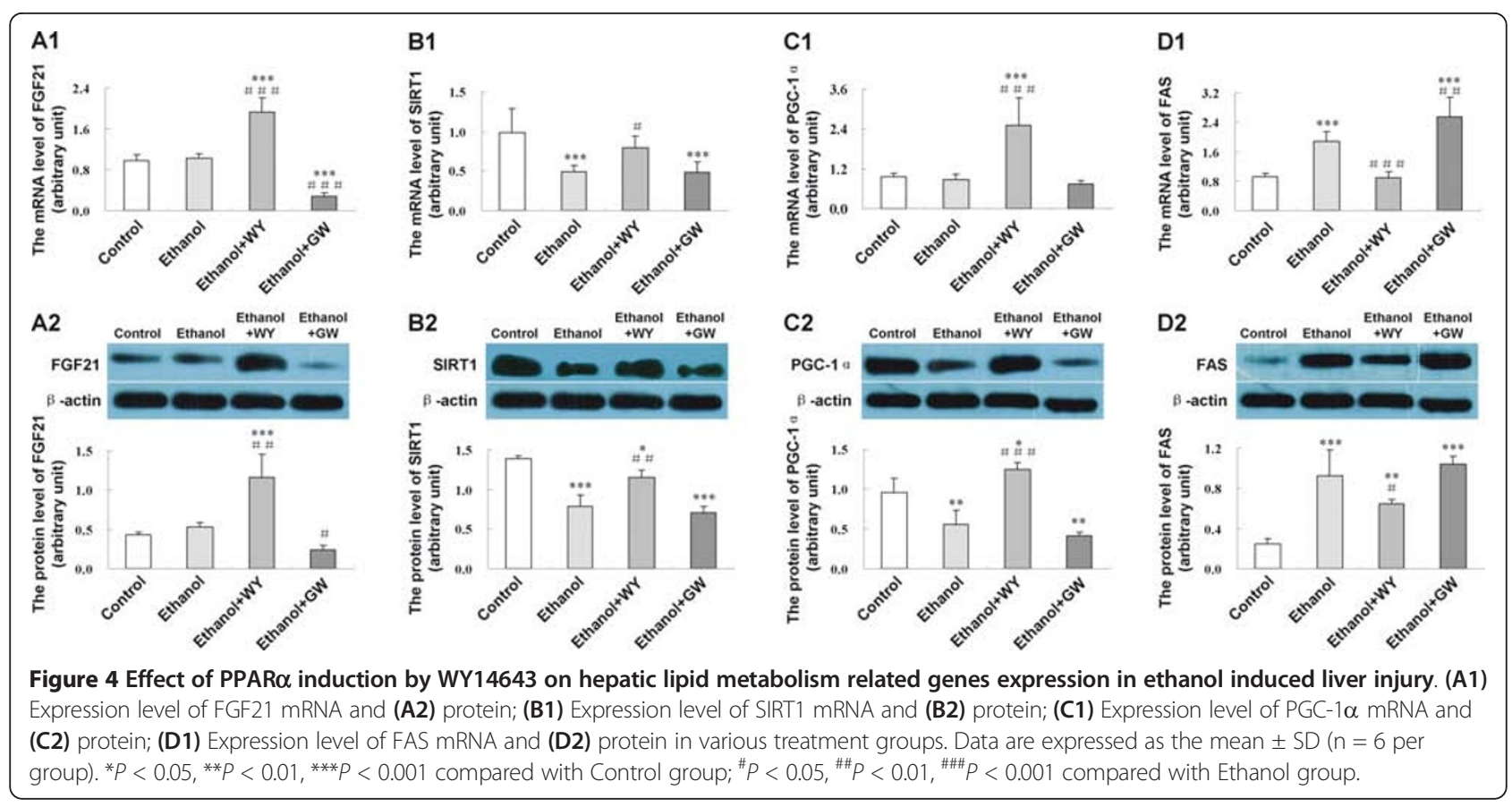

as up-regulation of key metabolic genes, including PGC$1 \alpha$ [21], a crucial metabolic mediator for coordinating gluconeogenesis and fatty acid oxidation in the liver [22-24]. In keeping with this observation, induction of PPAR $\alpha$ by
WY14643 in this study induced the hepatic expression of PGC-1 $\alpha$. Besides, SIRT1 repressed lipogenesis largely by attenuating the activity of SREBP-1 and its downstream gene FAS [20,25-28]. In this connection, downregulation

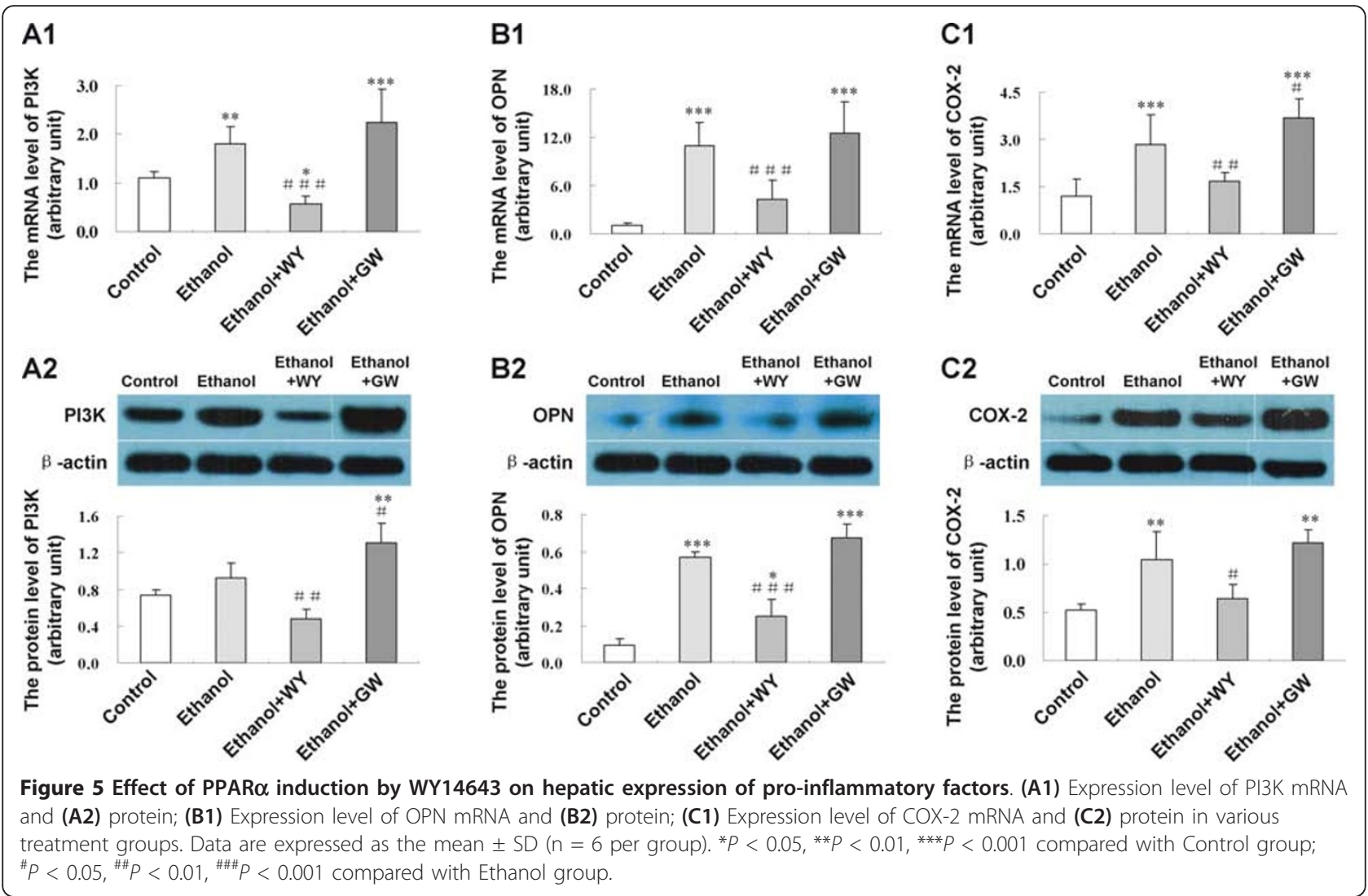




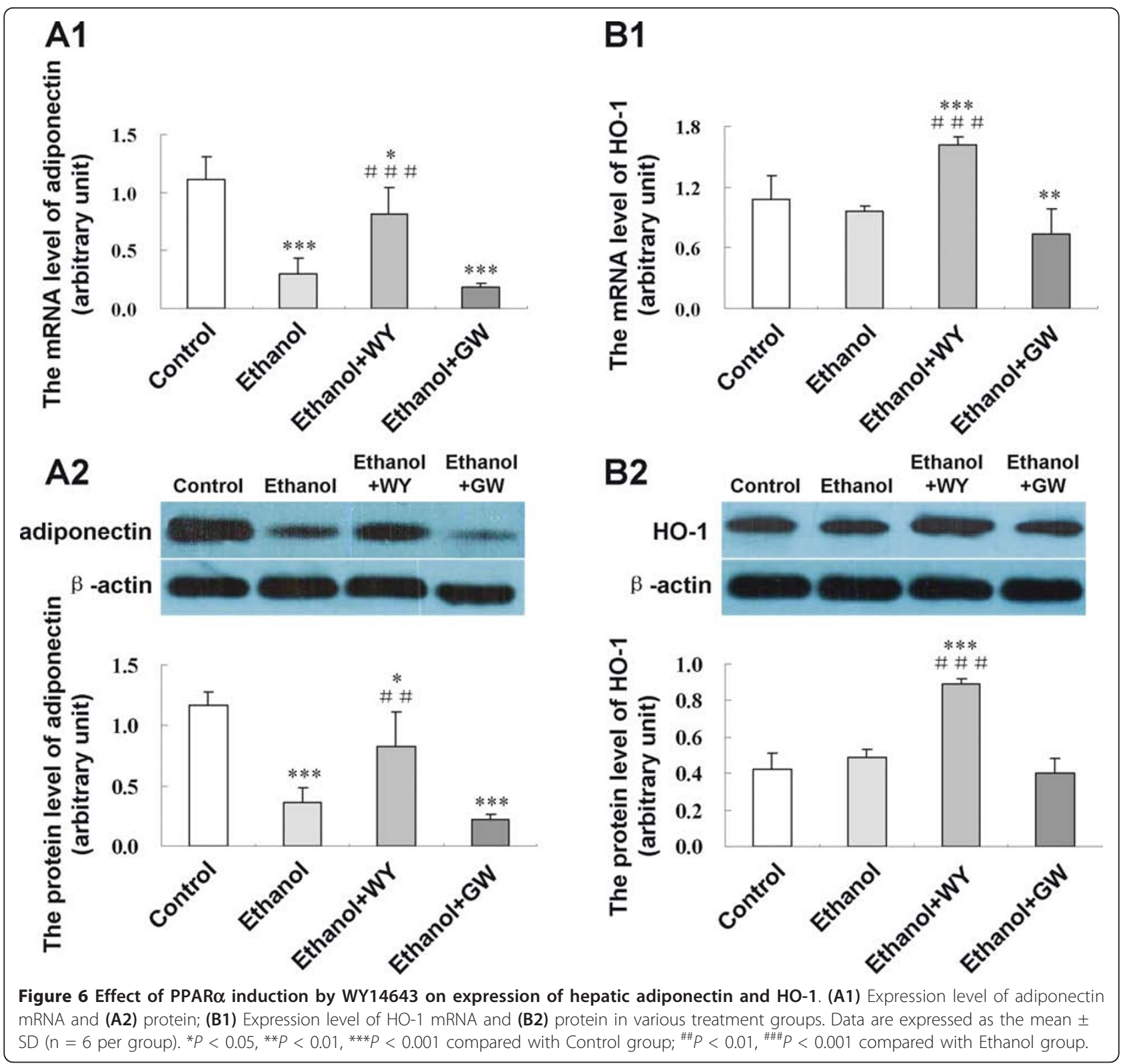

of FAS was demonstrated by WY14643 in this study. The SIRT1 axis has also emerged as a major signaling system in regulating adiponectin signaling in the lipid lowering action $[29,30]$. We also found that WY14643 increased adiponectin expression and down-regulated hepatic expression of PI3K in ethanol feeding mice, the latter has been reported to participate in the adipogenesis of human mesenchymal stem cells [31] and promote steatosis in cultured hepatocytes exposed to methionine-choline deficient (MCD) medium [32]. Collectively, the anti-steatosis effect of PPAR $\alpha$ may be partly related to up-regulation of its target genes CYP4A10 and CYP4A14, induction of SIRT1 axis by up-regulation of FGF21 and adiponectin expression, as well as down-regulation of FAS and PI3K expression, which ameliorated hepatic steatosis induced by ethanol consumption.

Ethanol treatment results in fatty acids accumulation in the liver. Fatty acids are substrate of cytochrome P450 2E1 (CYP 2E1). Induction of CYP 2E1 by fatty acids is a major source of reactive oxygen species (ROS), which evokes oxidative stress and mitochondrial damage in the hepatocytes, in turn worsens fatty acid $\beta$-oxidation disorder and hepatic steatosis. Furthermore, ROS activates and sensitizes Kupffer cells, which produce and release pro-inflammatory cytokines, triggering hepatic inflammatory response [11]. PI3K could induce expression of OPN, which increased ALT release by hepatocytes treated with MCD medium [32], indicating that PI3K and 
OPN were potentially involved in the development of hepatocyte damage. Elevated level of OPN, a chemoattractant molecule, correlated with neutrophil infiltration [4], and neutralizing antibody against OPN prevented neutrophil infiltration and ethanol induced liver injury [5]. Pro-inflammatory factor COX-2 was induced by ethanol as well. In our study, activation of PPAR $\alpha$ resulted in the inhibition of these key hepatic inflammatory regulators including $\mathrm{PI} 3 \mathrm{~K}, \mathrm{OPN}$ and COX-2 expression. In this regard, the anti-inflammatory effects of PPAR $\alpha$ in ethanol induced steatohepatitis may be related to inhibition of these pro-inflammatory factors.

Besides regulating lipid metabolism in the liver, adiponectin serves as an anti-inflammatory factor. Adiponectin prevents hepatic injury by inhibiting the synthesis and/or release of tumor necrosis factor- $\alpha$ (TNF- $\alpha$ ) [33]. HO-1 plays essential role in mediating the anti-inflammatory function of adiponectin. Importantly, after chronic ethanol exposure, induction of $\mathrm{HO}-1$ protects mice from the sensitizing effect of ethanol on LPS-stimulated TNF- $\alpha$ expression [34]. In addition, HO-1 and its down-stream mediator carbon monoxide could increase expression of anti-inflammatory cytokine interleukin-10 in macrophages [35]. In this study, HO-1 expression was up-regulated by PPAR $\alpha$ agonist. In keeping with our findings, Otterbein et al. reported that PPAR $\alpha$ could regulate HO- 1 transcription directly by binding to a PPAR-responsive element in the promoter regions of HO-1 [36]. Thus, PPAR $\alpha$ induced $\mathrm{HO}-1$ expression by itself and/or via increasing adiponectin expression may contribute to the amelioration of ethanol induced hepatic inflammatory response.

\section{Conclusions}

The present study showed a protective role of PPAR $\alpha$ in ethanol induced liver injury. Activation of PPAR $\alpha$ by WY14643 ameliorated hepatic steatosis through increasing lipids oxidation promoting genes CYP4A10, CYP4A14, FGF21, adiponectin, SIRT1 and PGC-1 $\alpha$ expression, and suppressing fatty acid synthesis promoting genes FAS and PI3K expression. Induction of PPAR $\alpha$ attenuated liver inflammatory response by repressing expression of proinflammatory cytokines PI3K, OPN and COX-2, as well as enhancing expression of anti-inflammatory factors adiponectin and HO-1. PPAR $\alpha$ agonist administration might serve as an effective therapeutic strategy for ALD.

\section{Materials and methods}

\section{Animals and treatments}

Eight-week-old male C57BL/6J mice with body weight between 20-25 g were obtained from the Experimental Animal Center of Chinese Academy of Medical Sciences, and were bred in a temperature-controlled animal facility with a $12 \mathrm{~h}$ light-dark cycle. They had free access to water and were allowed to adapt to their food and environment for 1 week before the start of the experiment. The mice were randomly divided into 4 groups (6 mice per group): 1) Control group: fed with non-alcohol control liquid diet; 2) Ethanol group: fed with $4 \%$ ethanol-containing LieberDeCarli liquid diet; 3) Ethanol plus WY14643 (Ethanol +WY) group: fed with ethanol liquid diet supplemented with WY14643 (50 mg/kg/d, Cayman Chemical, Ann Arbor, MI, CA); 4) Ethanol plus GW6471 (Ethanol+GW) group: fed with ethanol liquid diet plus GW6471 (10 mg/ kg, Tocris bioscience, Bristol, UK), three times/week, intraperitoneal injection. The ethanol concentration was raised gradually from $2 \%$ to $4 \%$ within 2 weeks, and then the mice were fed a $4 \%$ ethanol-containing Lieber-DeCarli liquid diet for 12 weeks. PPAR $\alpha$ agonist WY14643 and antagonist GW6471 were administrated in the last 2 weeks. Control mice were fed with the same volume of a control liquid diet prepared by replacing ethanol in the Lieber-DeCarli liquid diet with isocaloric maltose. Animals were sacrificed after overnight fasting at the end of experiment. Blood samples were collected from femoral artery for biochemical analysis. Livers were weighed, frozen or fixed in $10 \%$ formalin for histological analysis, fixed in $4 \%$ glutaraldehyde for electron microscopy, or snap-frozen in lipid nitrogen followed by storage at $-80^{\circ} \mathrm{C}$ in a freezer until required. All the protocols and procedures were performed following the guidelines of the Hebei Committee for Care and Use of Laboratory Animals and were approved by the Animal Experimentation Ethics Committee of the Hebei Medical University.

\section{Biochemical analysis}

Serum ALT and AST levels were measured by enzymatic method using an automatic biochemical analyzer (Olympus UA2700, Japan) according to the manufacturer's instructions.

\section{Histological analysis}

Haematoxylin and eosin stained paraffin-embedded liver sections ( $5 \mu \mathrm{m}$ thick) were scored as follows: (a) degree of steatosis ( $0 \leq 10 \%, 1=10-33 \%, 2=33-66 \%, 3 \geq 66 \%)$; (b) degree of hepatocyte ballooning ( $0=$ none, $1=$ mild and moderate, 2 = severe); (c) degree of necroinflammation ( 0 = none, 1 = mild, $2=$ moderate, $3=$ severe $)$ in accordance with a scoring system for ALD designed by Dominguez et al. [37].

\section{Transmission electron microscopy (TEM) examination}

The tissue fixed in $4 \%$ glutaraldehyde solution was postfixed in $1.5 \%$ osmium tetroxide solution, dehydrated, and fixed firmly into polybed resin. Micro-thin sections were performed and stained with lead citrate and uranyl acetate for a Hitachi model 7500 TEM examination. 


\section{Quantitative real-time reverse transcription polymerase chain reaction (qRT-PCR) analysis of hepatic $m R N A$ expression}

Total RNA was isolated from liver tissues using Trizol Reagent (Tiangen Biotech, Beijing, China) according to the manufacturer's instructions. The hepatic mRNA levels of PPAR $\alpha$, CYP4A10, CYP4A14, FGF21, SIRT1, PGC-1 $\alpha$, FAS, PI3K, OPN, COX-2, adiponectin and $\mathrm{HO}-1$ were determined by qRT-PCR using the ABI PRISM 7500 sequence detection system (Applied Biosystems, Foster, CA) with SYBR Green Reagent (Tiangen Biotech). Expression levels of the target genes were normalized against an endogenous reference gene glyceraldehydes 3-phosphate dehydrogenase (GAPDH). The specific primer sequences were listed in Table 1. All data were obtained using Sequence Detector Software (Applied Biosystems).

Table 1 Primer sequences used for real-time RT-PCR analysis

\begin{tabular}{|c|c|c|}
\hline Gene & Product length & Primer sequences \\
\hline \multirow[t]{2}{*}{ PPAR $\alpha$} & $149 \mathrm{bp}$ & 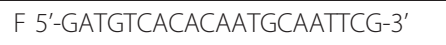 \\
\hline & & R 5'-GGTAGGCTTCGTGGATTCTCT-3' \\
\hline \multirow[t]{2}{*}{ CYP4A10 } & $64 \mathrm{bp}$ & F 5'-ACACTGCTCCGCTTCGAACT-3' \\
\hline & & F 5'-CAAGTCGGGCTAAGGGCA-3' \\
\hline \multirow[t]{2}{*}{ CYP4A14 } & $81 \mathrm{bp}$ & F 5'-AAGGCAGTGTTCAGTTGGATG-3' \\
\hline & & F 5'-GGCGAAAGAAAGTCAGGTTGT-3' \\
\hline \multirow[t]{2}{*}{ FGF21 } & $199 \mathrm{bp}$ & F 5'-CAGATGACGACCAAGACACTG-3' \\
\hline & & R 5'-TCAAAGTGAGGCGATCCATAG-3' \\
\hline \multirow[t]{2}{*}{ SIRT1 } & $197 \mathrm{bp}$ & F 5'-ACGCTGTGGCAGATTGTTATTA-3' \\
\hline & & R 5'-GCAAGGCGAGGCATAGATACC-3' \\
\hline \multirow[t]{2}{*}{ PGC-1 $\alpha$} & $109 \mathrm{bp}$ & F 5'-AGGAACAGCAGCAGAGACAAAT-3' \\
\hline & & R 5'-CTGGGGTCAGAGGAAGAGATAA-3' \\
\hline \multirow[t]{2}{*}{ FAS } & $193 \mathrm{bp}$ & F 5'-GGGTTCTAGCCAGCAGAGTCTA-3' \\
\hline & & R 5'- TGAGGTTGCTGTCGTCTGTAGT-3' \\
\hline \multirow[t]{2}{*}{ PI3K } & $198 \mathrm{bp}$ & F 5'- GCACGGCGATTACACTCTTAC-3' \\
\hline & & R 5'-TTGGACACTGGGTAGAGCAAC-3' \\
\hline \multirow[t]{2}{*}{ OPN } & $121 \mathrm{bp}$ & F 5'-GGTGATAGCTTGGCTTATGGAC-3' \\
\hline & & R 5'-CCTTAGACTCACCGCTCTTCAT-3' \\
\hline \multirow[t]{2}{*}{$\operatorname{cox}-2$} & $122 \mathrm{bp}$ & F 5'-GCCTTCTCCAACCTCTCCTACT-3' \\
\hline & & R 5'-ACCTITTCCAGCACTTCTITGG-3' \\
\hline \multirow[t]{2}{*}{ adiponectin } & $202 \mathrm{bp}$ & F 5'-CCAGTATCAGGAAAAGAATGTGG-3' \\
\hline & & R 5'-TGGTGTATGGGCTATGGGTAGT-3' \\
\hline \multirow[t]{2}{*}{$\mathrm{HO}-1$} & $427 \mathrm{bp}$ & F 5'-AACAAGCAGAACCCAGTCTATG-3' \\
\hline & & R 5'-TGAGCAGGAAGGCGTCTTA-3' \\
\hline \multirow[t]{2}{*}{ GAPDH } & $233 \mathrm{bp}$ & F 5'-GGTGAAGGTCGGTGTGAACG-3' \\
\hline & & R 5'-CTCGCTCCTGGAAGATGGTG-3' \\
\hline
\end{tabular}

Abbreviations: PPAR $\alpha$, peroxisome proliferator activated receptor alpha; CYP4A10, cytochrome P450 4A10; CYP4A14, cytochrome P450 4A14; FGF21, fibroblast growth factor 21; SIRT1, Sirtuin 1; PGC-1 $\alpha$, PPAR gamma coactivator $1 \alpha$; FAS, fatty acid synthase; PI3K, phosphatidylinositol 3-kinase; OPN, osteopontin; COX-2, cyclooxygenase 2; HO-1, heme oxygenase-1; GAPDH, glyceraldehyde-3- phosphate dehydrogenase.

\section{Western blot analysis of hepatic protein expression}

Total protein was extracted and concentration was measured by the Bradford method (DC protein assay; Bio-Rad, Hercules, CA). Equal amounts of protein $(100 \mu \mathrm{g} /$ well $)$ were loaded onto $12 \%$ SDS-PAGE for each sample and proteins were transferred onto equilibrated polyvinylidene difluoride membranes (Millipore Corporation, Billerica, MA) by electroblotting. The membranes were incubated with primary antibodies of PPAR $\alpha$, FGF21, SIRT1, PGC$1 \alpha$, FAS, PI3K, OPN, COX-2, adiponectin, HO-1, and $\beta$-actin (Santa Cruz Biotechnology, Santa Cruz, CA), respectively, overnight at $4^{\circ} \mathrm{C}$. Membranes were further incubated with secondary antibody for $1 \mathrm{~h}$ at room temperature. Proteins were detected by enhanced chemiluminescence (Santa Cruz Biotechnology). The amount of protein expression was corrected by that of $\beta$-actin in the same sample and the bands were quantified by scanning densitometry using the digital Kodak Gel Logic 200 (Carestream Molecular Imaging, Woodbridge, CT).

\section{Statistical analysis}

All data are expressed as mean \pm standard deviation (SD). Statistical analysis on the data was performed by one-way analysis of variance (ANOVA) or Kruskal-Wallis $H$ test, with the least significant difference- $t$ (LSD- $t$ ) test or mann-whitney $u$ test for post-hoc comparison using SPSS 13.0 (v. 13.0; SPSS Inc., Chicago, IL), and $P$-value below 0.05 was considered significant.

\section{List of Abbreviations}

ALD: alcoholic liver disease; ALT: alanine aminotransferase; AST: aspartic transaminase; COX-2: cyclooxygenase-2; CYP4A10: cytochrome P450 4A10; CYP4A14: cytochrome P450 4A14; FAS: fatty acid synthase; FFA: free fatty acid; FGF21: fibroblast growth factor 21; HO-1: heme oxygenase-1; OPN: osteopontin; PGC-1a: proliferators activated receptor gamma coactivator 1 alpha; PI3K: phosphatidylinositol 3-kinase; PPARa: Peroxisome proliferator activated receptor alpha; SIRT1: Sirtuin 1; SREBP-1: sterol regulatory element binding protein 1.

\section{Acknowledgements}

This work was funded by Wang Bao-en Foundation of Hepatic Fibrosis, Chinese foundation for hepatitis prevention and control, No. 2009009.

\section{Author details}

'Department of Traditional and Western Medical Hepatology, Third Hospital of Hebei Medical University, Shijiazhuang, China. ${ }^{2}$ Institute of Digestive Disease and Department of Medicine and Therapeutics, Li Ka Shing Institute of Health Sciences, The Chinese University of Hong Kong, Hong Kong.

\section{Authors' contributions}

YN designed the research; LK, WR, WL, SZ, HM, RW, YZ and WW performed the experiments; LK and YN analyzed data; YN, LK and JY wrote the paper. All authors read and approved the final manuscript.

\section{Competing interests}

The authors declare that they have no competing interests. The authors alone are responsible for the content and writing of the paper.

Received: 19 November 2011 Accepted: 30 December 2011 Published: 30 December 2011 


\section{References}

1. Tilg H, Diehl AM: Cytokines in alcoholic and nonalcoholic steatohepatitis. N Engl J Med 2000, 343:1467-1476.

2. Chaung WW, Jacob A, Ji Y, Wang P: Suppression of PGC-1alpha by Ethanol: Implications of Its Role in Alcohol Induced Liver Injury. Int J Clin Exp Med 2008, 1:161-170

3. Ji C, Chan C, Kaplowitz N: Predominant role of sterol response element binding proteins (SREBP) lipogenic pathways in hepatic steatosis in the murine intragastric ethanol feeding model. J Hepatol 2006, 45:717-724.

4. Banerjee A, Burghardt RC, Johnson GA, White FJ, Ramaiah SK: The temporal expression of osteopontin (SPP-1) in the rodent model of alcoholic steatohepatitis: a potential biomarker. Toxicol Pathol 2006, 34:373-384.

5. Banerjee A, Apte UM, Smith R, Ramaiah SK: Higher neutrophil infiltration mediated by osteopontin is a likely contributing factor to the increased susceptibility of females to alcoholic liver disease. J Pathol 2006, 208:473-485.

6. Spitzer JA, Zheng M, Kolls JK, Vande Stouwe C, Spitzer JJ: Ethanol and LPS modulate NF-kappaB activation, inducible NO synthase and COX-2 gene expression in rat liver cells in vivo. Front Biosci 2002, 7:a99-108.

7. Crabb DW, Galli A, Fischer M, You M: Molecular mechanisms of alcoholic fatty liver: role of peroxisome proliferator-activated receptor alpha. Alcohol 2004, 34:35-38.

8. Donohue TM Jr: Alcohol-induced steatosis in liver cells. World J Gastroenterol 2007, 13:4974-4978

9. Aoyama T, Peters JM, Iritani N, Nakajima T, Furihata K, Hashimoto T, Gonzalez FJ: Altered constitutive expression of fatty acid-metabolizing enzymes in mice lacking the peroxisome proliferator-activated receptor alpha (PPARalpha). J Biol Chem 1998, 273:5678-5684.

10. Watanabe K, Fujii H, Takahashi T, Kodama M, Aizawa Y, Ohta Y, Ono T, Hasegawa G, Naito M, Nakajima T, Kamijo Y, Gonzalez FJ, Aoyama T: Constitutive regulation of cardiac fatty acid metabolism through peroxisome proliferator-activated receptor alpha associated with agedependent cardiac toxicity. J Biol Chem 2000, 275:22293-22299.

11. Moriya T, Naito H, Ito Y, Nakajima T: "Hypothesis of Seven Balances": Molecular Mechanisms behind Alcoholic Liver Diseases and Association with PPAR. J Occup Health 2009, 51:391-403.

12. Shulman Al, Mangelsdorf DJ: Retinoid X Receptor heterodimers in the metabolic syndrome. N Engl J Med 2005, 353:604-615.

13. Bocos C, Göttlicher M, Gearing K, Banner C, Enmark E, Teboul M, Crickmore A, Gustafsson JA: Fatty acid activation of peroxisome proliferator-activated receptor (PPAR). J Steroid Biochem Mol Biol 1995, 53:467-473.

14. Issemann I, Green S: Activation of a member of the steroid hormone receptor superfamily by peroxisome proliferators. Nature 1990, 347:645-650.

15. Patsouris D, Reddy JK, Müller M, Kersten S: Peroxisome ProliferatorActivated Receptor a Mediates the Effects of High-Fat Diet on Hepatic Gene Expression. Endocrinology 2006, 147:1508-1516.

16. Badman MK, Pissios P, Kennedy AR, Koukos G, Flier JS, Maratos-Flier E: Hepatic fibroblast growth factor 21 is regulated by PPARa and is a key mediator of hepatic lipid metabolism in ketotic states. Cell Metab 2007, 5:426-437

17. Inagaki $T$, Dutchak $P$, Zhao G, Ding $X$, Gautron $L$, Parameswara V, Li $Y$, Goetz R, Mohammadi M, Esser V, Elmquist JK, Gerard RD, Burgess SC, Hammer RE, Mangelsdorf DJ, Kliewer SA: Endocrine regulation of the fasting response by PPAR-mediated induction of fibroblast growth factor 21. Cell Metab 2007, 5:415-425.

18. Kharitonenkov A, Shiyanova TL, Koester A, Ford AM, Micanovic R, Galbreath EJ, Sandusky GE, Hammond LJ, Moyers JS, Owens RA, Gromada J, Brozinick JT, Hawkins ED, Wroblewski VJ, Li DS, Mehrbod F, Jaskunas SR, Shanafelt AB: FGF-21 as a novel metabolic regulator. J Clin Invest 2005 115:1627-1635.

19. Lieber CS, Leo MA, Wang X, Decarli LM: Effect of chronic alcoho consumption on hepatic SIRT1 and PGC-1alpha in rats. Biochem Biophys Res Commun 2008, 370:44-48.

20. You M, Liang X, Ajmo JM, Ness GC: Involvement of mammalian sirtuin 1 in the action of ethanol in the liver. Am J Physiol Gastrointest Liver Physiol 2008, 294:G892-898.

21. Chau MD, Gao J, Yang Q, Wu Z, Gromada J: Fibroblast growth factor 21 regulates energy metabolism by activating the AMPK-SIRT1-PGC-1a pathway. Proc Natl Acad Sci USA 2010, 107:12553-12558.
22. Handschin C, Spiegelman BM: Peroxisome proliferator-activated receptor y coactivator 1 coactivators, energy homeostasis, and metabolism. Endocr Rev 2006, 27:728-735.

23. Puigserver $P$, Spiegelman BM: Peroxisome proliferator-activated receptorgamma coactivator 1 alpha (PGC-1 alpha): transcriptional coactivator and metabolic regulator. Endocr Rev 2003, 24:78-90.

24. Lin J, Handschin C, Spiegelman BM: Metabolic control through the PGC-1 family of transcription coactivators. Cell Metab 2005, 1:361-370.

25. Cantó C, Auwerx J: PGC-1alpha, SIRT1 and AMPK, an energy sensing network that controls energy expenditure. Curr Opin Lipidol 2009, 20:98-105.

26. Cantó C, Gerhart-Hines Z, Feige JN, Lagouge M, Noriega L, Milne JC, Elliott PJ, Puigserver $P$, Auwerx J: AMPK regulates energy expenditure by modulating NAD+ metabolism and SIRT1 activity. Nature 2009, 458:1056-1060.

27. Purushotham A, Schug TT, Xu Q, Surapureddi S, Guo X, Li X: Hepatocytespecific deletion of SIRT1 alters fatty acid metabolism and results in hepatic steatosis and inflammation. Cell Metab 2009, 9:327-338.

28. Suchankova G, Nelson LE, Gerhart-Hines Z, Kelly M, Gauthier MS, Saha AK, Ido $Y$, Puigserver $P$, Ruderman NB: Concurrent regulation of AMPactivated protein kinase and SIRT1 in mammalian cells. Biochem Biophys Res Commun 2009, 378:836-841.

29. Rogers CQ, Ajmo JM, You M: Adiponectin and alcoholic fatty liver disease. IUBMB Life 2008, 60:790-797.

30. You M, Rogers CQ: Adiponectin: a key adipokine in alcoholic fatty liver. Exp Biol Med (Maywood) 2009, 234:2850-2959.

31. Yu W, Chen Z, Zhang J, Zhang L, Ke H, Huang L, Peng Y, Zhang X, Li S, Lahn BT, Xiang AP: Critical role of phosphoinositide 3-kinase cascade in adipogenesis of human mesenchymal stem cells. Mol Cell Biochem 2008, 310:11-18

32. Sahai A, Pan X, Paul R, Malladi P, Kohli R, Whitington PF: Roles of phosphatidylinositol 3-kinase and osteopontin in steatosis and aminotransferase release by hepatocytes treated with methioninecholine-deficient medium. Am J Physiol Gastrointest Liver Physiol 2006, 291: G55-62.

33. Masaki T, Chiba S, Tatsukawa H, Yasuda T, Noguchi H, Seike M, Yoshimatsu H: Adiponectin protects LPS-induced liver injury through modulation of TNF-alpha in KK-Ay obese mice. Hepatology 2004, 40:177-184.

34. Mandal P, Park PH, McMullen MR, Pratt BT, Nagy LE: The Anti-Inflammatory Effects of Adiponectin Are Mediated via a Heme Oxygenase-1 Dependent Pathway in Rat Kupffer Cells. Hepatology 2010, 51:1420-1429.

35. Otterbein LE, Soares MP, Yamashita K, Bach FH: Heme oxygenase-1: unleashing the protective properties of heme. Trends Immunol 2003, 24:449-455.

36. Lin H, Yu CH, Jen CY, Cheng CF, Chou Y, Chang CC, Juan SH: Adiponectinmediated heme oxygenase- 1 induction protects against iron-induced liver injury via a PPARa dependent mechanism. Am J Pathol 2010 177:1697-1709.

37. Dominguez M, Rincón D, Abraldes JG, Miquel R, Colmenero J, Bellot $P$ García-Pagán JC, Fernández R, Moreno M, Bañares R, Arroyo V, Caballería J, Ginès $P$, Bataller R: A new scoring system for prognostic stratification of patients with alcoholic hepatitis. Am J Gastroenterol 2008, 103:2747-2756.

doi:10.1186/1476-511X-10-246

Cite this article as: Kong et al:: Activation of peroxisome proliferator activated receptor alpha ameliorates ethanol induced steatohepatitis in mice. Lipids in Health and Disease 2011 10:246. 